\title{
Editorial
}

\section{Complexity in Medical Informatics}

\author{
Panagiotis Vlamos $\mathbb{D}^{1},{ }^{1}$ Ilias Kotsireas, ${ }^{2}$ and Dimitrios Vlachakis $\mathbb{D}^{3}$ \\ ${ }^{1}$ Bioinformatics and Human Electrophysiology Laboratory, Department of Informatics, Ionian University, Greece \\ ${ }^{2}$ Department of Physics and Computer Science, Wilfrid Laurier University, Ontario, Canada \\ ${ }^{3}$ Department of Biotechnology, Agricultural University of Athens, Greece
}

Correspondence should be addressed to Panagiotis Vlamos; vlamos@ionio.gr

Received 4 December 2018; Accepted 26 May 2019; Published 1 July 2019

Copyright (C) 2019 Panagiotis Vlamos et al. This is an open access article distributed under the Creative Commons Attribution License, which permits unrestricted use, distribution, and reproduction in any medium, provided the original work is properly cited.

In the era of big data and personalized medicine, the need to develop alternative methods for analyzing large volumes of data generated by clinical IoT platforms has formed. Big data analysis utilizes several data mining algorithms to determine patterns and connections in large databases [1].

Research studies use numerous data mining methodologies (i.e., machine learning algorithms). Genetic algorithm (GA) is a random optimization method derived from Darwin's principle of survival of the fittest in natural genetics [2]. GA is a metaheuristic method used in data mining applications such as feature selection and classification. According to Goldberg, 2012, the main idea behind GAs was robustness. Since their development, GAs proved to be a robust search tool. The fact that their use is not restricted by the search space is thought to be one of their biggest advantages [3]. GAs are of interest since they are not only very potent tools but also widely applicable ones. Among other medical areas, GAs are particularly useful for neurology, pharmacotherapy, and healthcare management [4]. GAs can also be used to train artificial neural networks (ANNs). A study showed that GA, in combination with the Levenberg-Marquardt backpropagation (LM) algorithm, was the best algorithm for ANN training, with $96.5 \%$ general success [5].

Another prevailing data processing technique is neural networks (NNs), and as their name suggests, they compare to the way the brain processes information. They are (usually) a nonlinear statistical tool, used to model complex relationships between data inputs and outputs or to establish data patterns. Current uses of neural networks include image analysis, signal processing, and laboratory medicine.
Regarding the field of laboratory medicine, researchers aim to improve scientific procedures by minimizing errors and decrease the workload of the laboratory staff, while maintaining/improving the safety of patients.

Figure 1 aptly describes the workflow of image processing. NNs play a significant role in each step. For step 1 (preprocessing), NNs can be utilized to solve the following problems: (1) optimization, (2) approximation, and (3) mapping. For data reduction (step 2), NNs aid in obtaining an image compression rate as high as possible. NNs are also trained (i) to implement feature extraction and (ii) pixel-based segmentation (step 3) and (iii) to locate objects based on pixel data (step 4). For step 5, NNs' possible uses include object classification (e.g., chromosomes) and camera image analysis. For optimization (step 6), NNs were employed for tasks regarding segmentation and recognition [6].

The complexity and difficulties in analyzing big data pushed science to search for new and highly efficient solutions to the said issue. These aforementioned approaches are utilized for the extraction of useful information for data analysis and decision-making purposes. The aim of this special issue is to examine the applicability of novel techniques and especially the accuracy of the neural network methodologies and genetic algorithms in multivariate analysis of clinical data.

Technological interfaces related to networking issues, such as data mining, machine and deep learning, and intelligent decision support systems, can be exploited to address challenges regarding the improvement of patient's safety, the enhancement of care outcomes, the promotion of a patientcentered care, the facilitation of translational research, the 


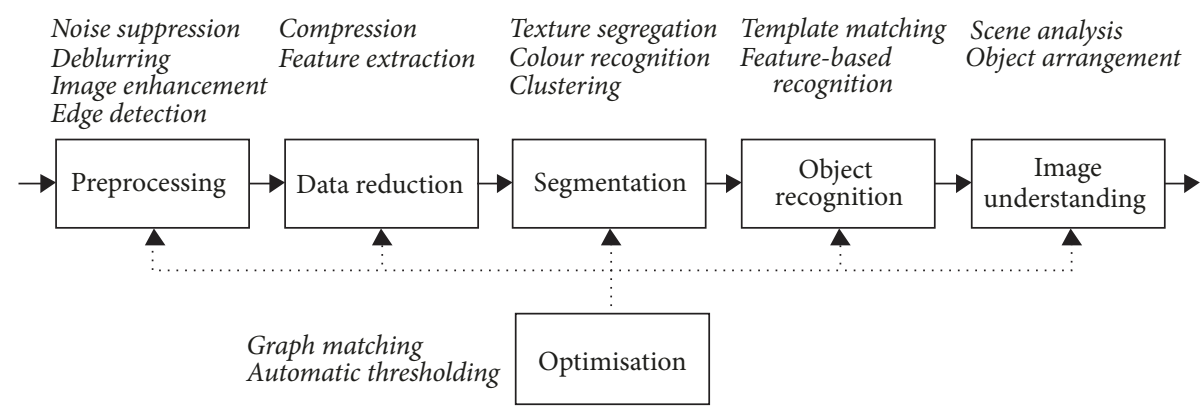

FIGURE 1: Image processing workflow (figure taken from [6]).

activation of precision medicine, and the improvement of education and skills in health informatics.

The topics of the accepted articles include but are not limited to the following: machine and deep learning approaches for health data; data mining and knowledge discovery in healthcare; clinical decision support systems; applications of the genetic algorithm in disease screening, diagnosis, and treatment planning; neurofuzzy system based on genetic algorithm for medical diagnosis and therapy support systems; applications of AI in healthcare; applications of artificial neural networks in medical science; electronic medical record and missing data; network and disease modeling (using administrative data); and health analytics and visualization.

\section{Conflicts of Interest}

The authors declare that there are no conflicts of interest regarding the publication of this special issue.

\section{Panagiotis Vlamos Ilias Kotsireas Dimitrios Vlachakis}

\section{References}

[1] C. H. Lee and H. Yoon, "Medical big data: promise and challenges," Kidney Research and Clinical Practice, vol. 36, no. 1, pp. 3-11, 2017.

[2] T. Jun-shan, H. Wei, and Q. Yan, "Application of genetic algorithm in data mining," in Proceedings of the 2009 First International Workshop on Education Technology and Computer Science, pp. 353-356, Wuhan, Hubei, China, March 2009.

[3] D. Goldberg, Genetic Algorithms in Search, Optimization, and Machine Learning, Addison-Wesley, Boston, Mass, USA, 2012.

[4] A. Ghaheri, S. Shoar, M. Naderan, and S. S. Hoseini, "The applications of genetic algorithms in medicine," Oman Medical Journal, vol. 30, no. 6, pp. 406-416, 2015.

[5] S. Koçer and M. R. Canal, "Classifying epilepsy diseases using artificial neural networks and genetic algorithm," Journal of Medical Systems, vol. 35, no. 4, pp. 489-498, 2011.

[6] M. Egmont-Petersen, D. de Ridder, and H. Handels, "Image processing with neural networks-a review," Pattern Recognition, vol. 35, no. 10, pp. 2279-2301, 2002. 


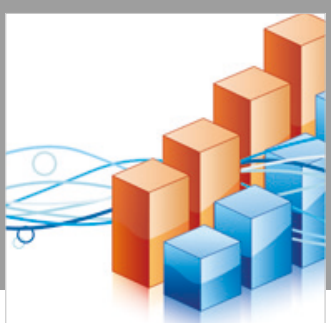

Advances in

Operations Research

\section{-n-m}
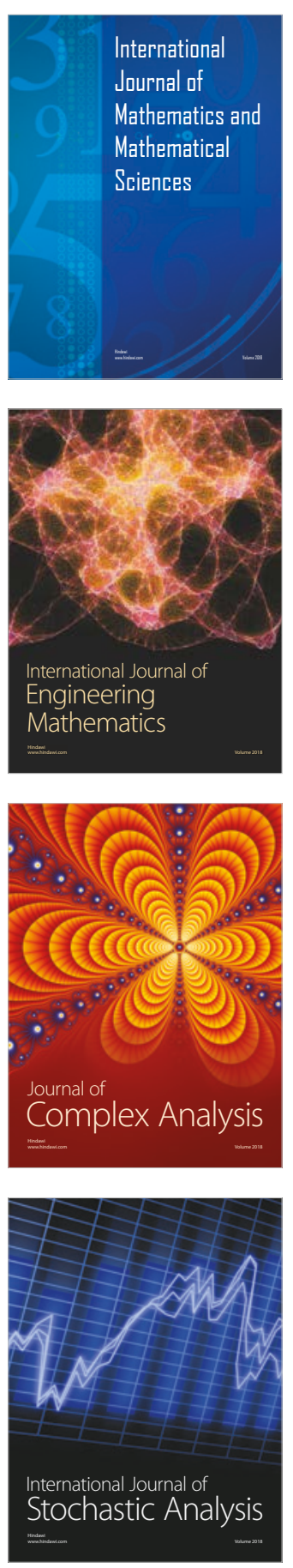
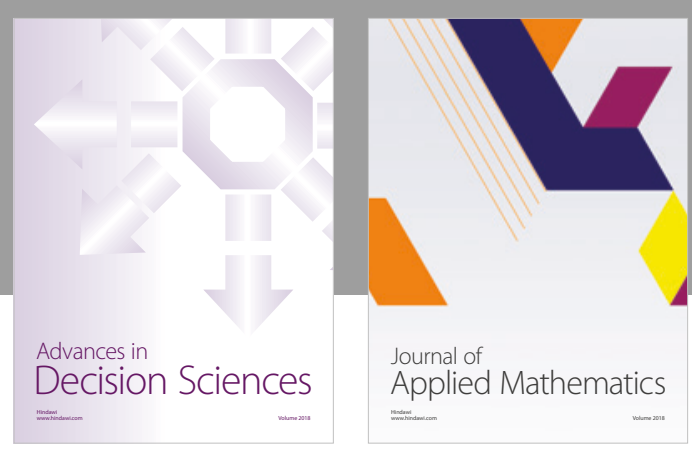

Journal of

Applied Mathematics
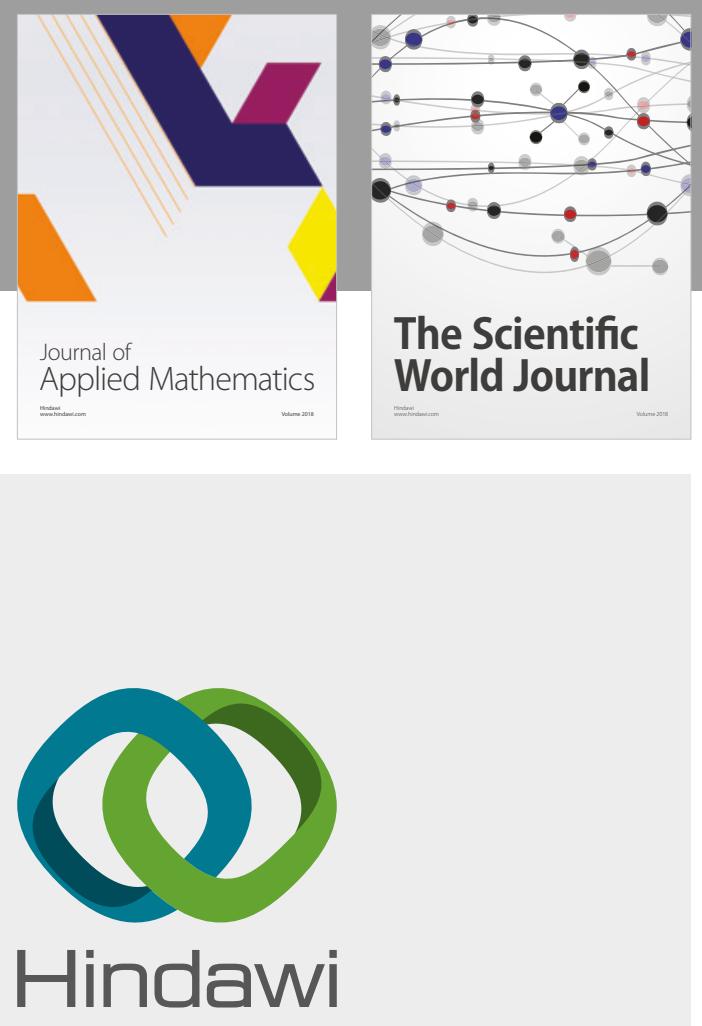

Submit your manuscripts at

www.hindawi.com

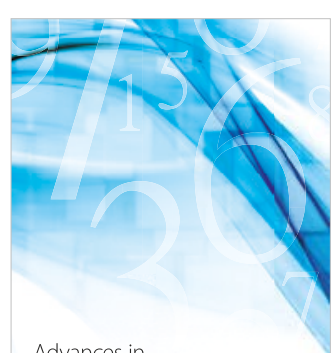

Advances in
Numerical Analysis
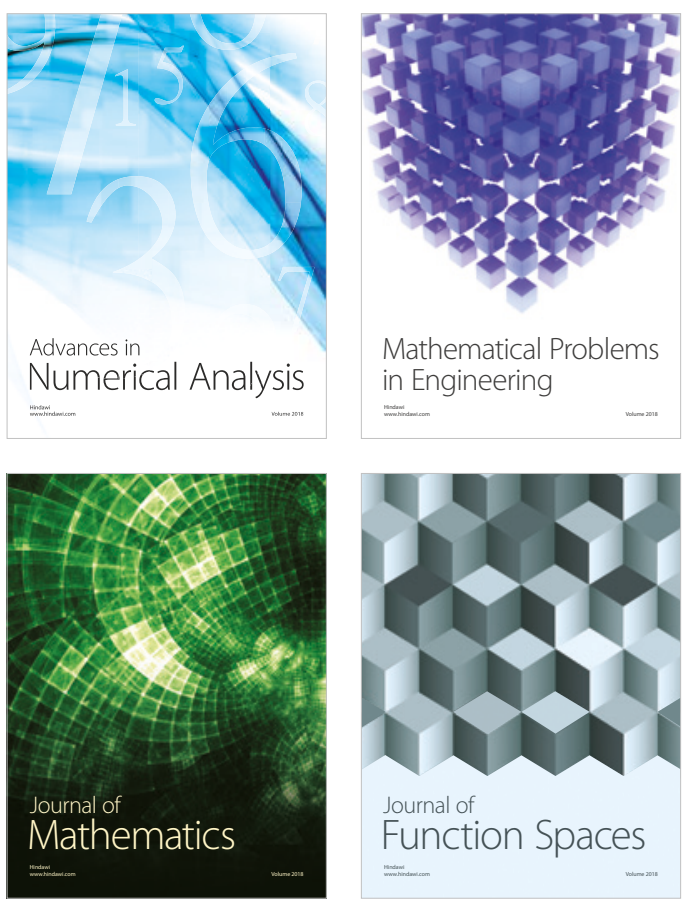

Mathematical Problems in Engineering

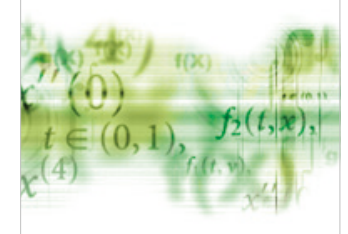

International Journal of

Differential Equations

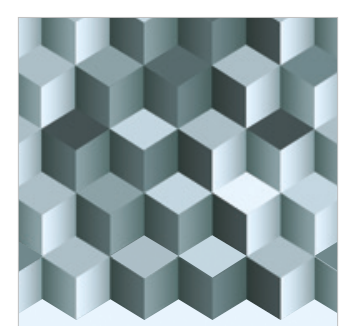

Journal of

Function Spaces
The Scientific

World Journal

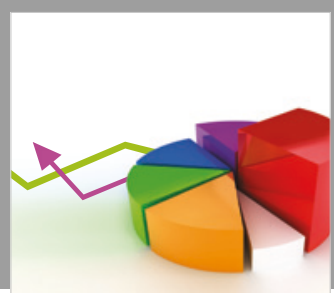

Journal of

Probability and Statistics
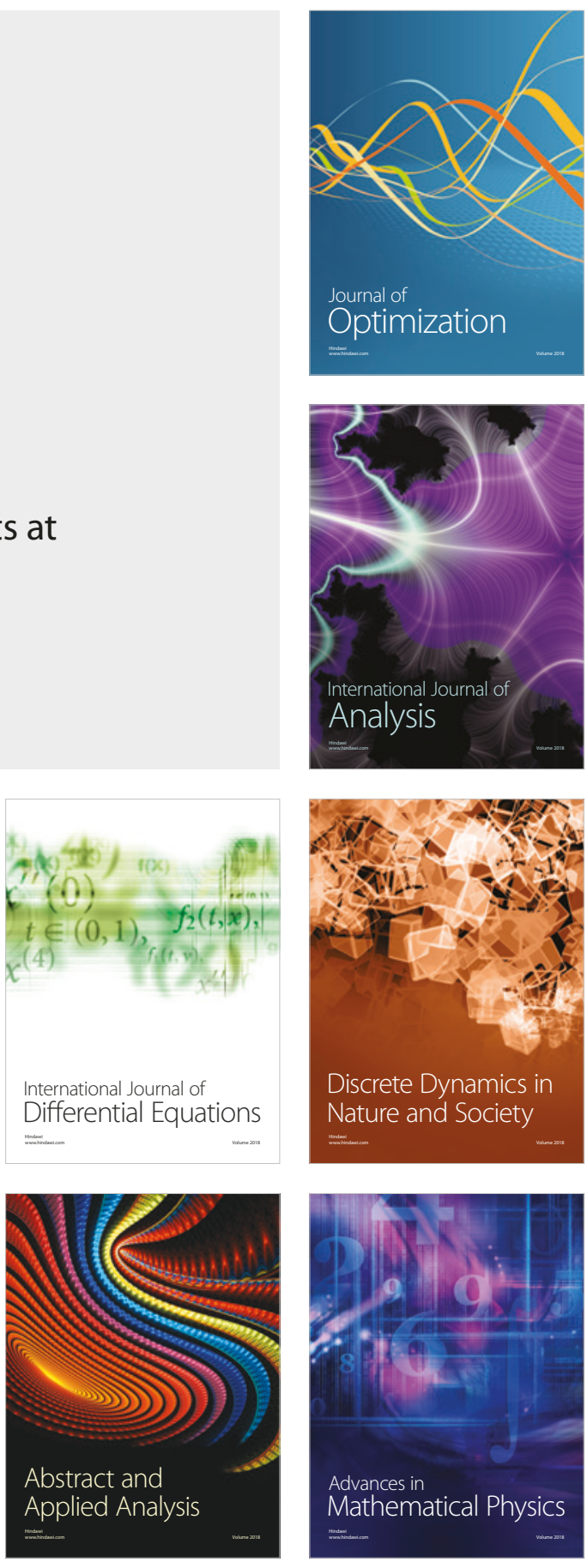